\title{
El rol de la televisión en la socialización politica de los niños: resultados preliminares
}

\author{
Enrique Huerta* \\ Berenice Bañuelos \\ Alejandra Rodríguez*
}

Instituto Tecnológico de Estudios Superiores de Monterrey, Campus Monterrey

\author{
Sandra Iliana ${ }^{* *}$ \\ Carmen Gómez \\ Universidad Autónoma de Tamaulipas
}

Este trabajo reporta hallazgos preliminares del análisis de 60 entrevistas grupales semiestructuradas $(\mathrm{n}=180)$ realizadas en marzo de 2006 en Coahuila, Nuevo León y Tamaulipas. Las entrevistas fueron realizadas para explorar la relación entre la televisión y la socialización política de los niños. Específicamente, el análisis pone atención a la relación entre los procesos de comunicación interpersonal; la exposición a los contenidos televisivos; las concepciones, los valores y las orientaciones políticas, y las prácticas y competencias cívicas. Un punto de partida para este estudio es que la visibilidad de la esfera pública en pleno proceso electoral permite que los niños tengan una visión más cercana de los procesos políticos. Los hallazgos han permitido el diseńo de un modelo estructural que será confrontado en una fase posterior del estudio.

This paper reports the preliminary findings from the analysis of 60 group interviews made in March (2006) in the States of Coahuila, Nuevo León

* Centro de Investigación en Comunicación e Información, Instituto Tecnológico y de Estudios Superiores de Monterrey

** Universidad Autónoma de Tamaulipas. 
and Tamaulipas. The interviews are meant to explore the connection between television and the political socialization of children. Specifically, the research pays special attention to the relation between the process of interpersonal communication and the exposition to the television contents and the concepts, values and the political orientation shown by the children under study and their civic competences and behavior. A necessary point of departure for this study is the political moment that pervades during elections year in Mexico, such as the present, allows the children under study to more sensitive to the political process. The findings have led to the design a conceptual model that will be compared to empirical evidence in a later phase of this study.

\section{Antecedentes}

La cultura política actual de los mexicanos tiene sus bases en los procesos de socialización de que fueron objetos durante su infancia, adolescencia y juventud. Entre los agentes participantes (familia, amigos, escuela), los medios de comunicación, y en particular la televisión, han adquirido una importancia fundamental en el actual proceso electoral.

El contacto de la ciudadanía con la política, los funcionarios y el gobierno ocurre necesariamente a través de procesos mediados. Podría creerse que los nińos no se exponen a mensajes políticos ni participan en un proceso de socialización política significativo. Sin embargo, la evidencia empírica es cada vez mayor en relación a la gran cantidad de información política con la que interactúan los niños en su vida cotidiana (López Gómez, 2003, Buckingham, 2000). El 60 por ciento de los niños de Monterrey y Juchitán, por ejemplo, reportaban en una investigación reciente (López Gómez, 2003, p. 55) sentarse a ver televisión de 3 a 7 días a la semana cuando sus papás veían noticias en la televisión y entre el 76 y el $80 \%$ de ellos afirmaba que se hablaba de política en sus casas.

Asimismo, otros estudios (Eveland, 1998) apuntan a que para cuando terminan la primaria, los niños han completado las competencias cognitivas relativas a la formación de ciudadanía, competencias predictoras de comportamiento político futuro (p.700). De acuerdo con Gunter y McAleer (1997), los nińos empiezan a tomar conciencia acerca de los asuntos políticos durante los primeros ańos de escuela y son capaces no sólo de articular ciertos argumentos al respecto, sino de identificar figuras e instituciones políticas. Según ellos, si bien la afiliación a ciertos partidos políticos puede ser formada por la familia,

274 Enrique Huerta, Berenice Bañuelos, Alejandra Rodríguez, Sandra Ileana, Carmen Gómez 
el conocimiento político se relaciona con el uso de los medios masivos y, especialmente, con el consumo de noticias y de temas actuales (p.61).

La socialización política se puede definir como el proceso por el que los individuos desarrollan concepciones de sí mismos y su mundo, así como del mundo político, incluyendo sus experiencias directas, juicios e inferencias sobre el conocimiento que en ese momento poseen (Weintraub y Pinkleton, 2001). A través del estudio de la socialización política es posible conocer las condiciones actuales del interés en los asuntos públicos, y vislumbrar en qué medida se está formando a una nueva generación de ciudadanos, concientes y participativos.

La investigación respecto a los procesos de socialización política coincide en señalar la influencia de la interacción entre la comunicación interpersonal y la exposición a contenidos políticos en los medios de comunicación masiva (Kiousis, Devitt y Wu, 2005). Variables como grado de educación de los padres, así como frecuencia y calidad de la exposición de los padres a contenidos políticos, se relacionan fuertemente con la frecuencia de la discusión, conocimiento e interés de temas políticos en los niños, así como con competencias cívicas (Eveland, 1998; Tapia, 2003; Weintraum y Pinkleton, 2001).

Dado el énfasis que las culturas occidentales han puesto a la política como competencia electoral y a la protección de los derechos de los niños, la temporalidad en que se estudian los fenómenos de socialización política resulta relevante. Varios autores observan distintas actitudes derivadas de la socialización política como el cinismo, la apatía, la ignorancia, preocupación política, falta de confianza en el gobierno (Buckingham, 2000), entusiasmo, aburrimiento, desinterés en cuestiones políticas, (Tapia, 2003) o el escepticismo (Weintraub y Pinkleton, 2001).

Sin embargo, otros estudios apuntan a que una campaña política es un marco idóneo para la observación de estos fenómenos, particularmente en niños. Sears y Valentino (1998) diseñaron un estudio en tres etapas. Realizaron un estudio longitudinal entrevistando a 366 pares de padres e hijos un año antes de una campaña política, justo después de la campaña y un año después. Sus hallazgos apuntan a que una campaña política "cristaliza" las actitudes y predisposiciones políticas de los niños y adolescentes, en la medida en que se exponen a los contenidos políticos de los medios y se enriquece con la conversación de los padres. Sus hallazgos sugieren que el grado de conocimiento político de los niños y adolescentes también depende del grado de conocimiento político de los padres (Sears y Valentino, 1998). Dado que los medios establecen agendas públicas de discusión, los niños pueden recordar las discusiones recientes y 
tomar partido desde sus propias formaciones de valores. Como se ha dicho arriba, la evidencia sugiere que estas convicciones los acompañarán a lo largo de su vida, aun si no están presentes en el primer plano de lo consciente la mayor parte del tiempo.

El estudio de la socialización política en los niños no es aséptico ni imparcial. Un punto de partida es que la formación de ciudadanía es valiosa para la sociedad en su conjunto. En primera instancia, supone conocer cuáles son, si hay, los valores y concepciones de la política en los niños. Además del conocimiento y el tipo de exposiciones que configuran su idea de aspectos políticos, se han estudiado las concepciones a la autoridad, la percepción del país, las representaciones de la democracia (Tapia, 2003), también conocimientos, virtudes cívicas y capital social (Buckingham, 2000). En segunda instancia, supone conocer cuáles son, si hay, las predisposiciones y competencias cívicas para la participación política. Kiousis, Devitt y XuWu (2005) estudiaron la fuerza de la opinión y la predisposición política en cuanto a las tendencias a uno u otro partido. Sus estudios de tres ciudades del suroeste de los Estados Unidos apoyaron un modelo estructural de covarianza que muestra que el tamaño de las redes de discusión predice la fortaleza de la ideología política de los niños y adolescentes. Mortimore y Tyrrel (2004) se interesaron en observar intención de voto, participación social y niveles de confianza en los actores políticos. En una muestra de 914 niños, encontraron que los niños mostraron un buen grado de conocimiento sobre política y que sus opiniones políticas parecían asociarse a las de sus padres. Una tercera instancia supondría la utilización de los factores que inciden en la socialización política, particularmente los medios de comunicación, para incrementar los niveles de conocimiento, interés, predisposiciones y competencias cívicas.

En México, la investigación sobre socialización política, medios de comunicación y niños no es reciente. Si bien no abundan los datos al respecto, ya desde los setenta, Segovia (1975) prestó atención al fenómeno. Aún cuando los cambios políticos y sociales hacen lucir ahora anticuadas algunas partes de su estudio, y lamentablemente no surgió de él una línea de investigación que se sostuviera, este pionero del tema es aún relevante porque sus datos permiten desde entonces saber que los niños mexicanos prestan atención a los temas políticos, sin el "cinismo" que Buckingham (2000) por ejemplo, encuentra en jóvenes anglosajones.

Este dato ha sido nuevamente hallado por estudios recientes como los de Tapia (2003) y López (2003). En sus estudios de niños del Distrito Federal,

276 Enrique Huerta, Berenice Bañuelos, Alejandra Rodriguez, Sandra Ileana, Carmen Gómez 
Oaxaca y Nuevo León, las investigadoras encontraron que los niños se mostraron interesados por temas políticos, particularmente aquellos de niveles socioeconómicos altos, quienes siempre tienen algún ejemplo cercano de líderes políticos de referencia (López, 2003). A diferencia del consenso internacional, Tapia (2003) encontró que los niños no reportan como su primera fuente de discusión de estos temas a los padres, sino a otros miembros de la familia o a amigos. Un dato similar fue reportado por Ibarra (2003). En su estudio de los niños de Guadalajara reportó que los niños decían comunicarse más de estos temas con sus maestros que con sus padres, aunque los datos se mantuvieron a nivel descriptivo y no permiten inferencias de comportamiento.

Debido a la influencia del Instituto Federal Electoral en los contenidos educativos y la agenda de intereses de investigación, en México ha importado conocer cómo los procesos de comunicación interpersonal y masiva influyen el conocimiento de los valores de la democracia y las predisposiciones y competencias políticas de los nińos (Ibarra, 2003; Tapia, 2003). Estos valores y competencias cívicas son particularmente relevantes en un tiempo de formación de nuevos ciudadanos que producirán una sociedad democrática. Sin embargo, los datos descriptivos con que se cuenta están lejos de ser conclusivos. Si bien son valiosos, sólo permiten hasta ahora elaborar hipótesis de comportamiento de los fenómenos. Por ejemplo, los estudios de Ibarra (2003), López (2003) y Tapia (2003) coincidieron en tiempos pero no se consultaron entre sí ni reportaron evidencia de pruebas de confiabilidad y validez que permitieran el re-uso de sus instrumentos con conocimiento de sus características psicométricas. Ante ello, resulta necesario el establecimiento de modelos e instrumentos válidos y confiables que permitan realizar estudios conclusivos en la materia.

Ante ello, se propone un estudio cualitativo que permita la exploración de cuatro ejes que, de acuerdo con la literatura, parecen cruciales al estudio de los procesos de socialización política, consumo de medios de comunicación y niños:

1. Las redes de comunicación interpersonal y las instituciones que colaboran en los procesos de socialización política.

2. La exposición a contenidos televisivos relacionados con la política, relación específica de la televisión y otros medios de comunicación en los procesos de socialización política de los niños.

3. El plano simbólico de las concepciones, los valores y las orientaciones políticas.

El rol de la televisión en la socialización politica de los niños: resultados preliminares $\bullet 277$ 
4. Las prácticas políticas y las competencias cívicas, para ir del plano conceptual al de la intención de participar, de la puesta en marcha de sus competencias cívicas.

\section{MÉTODO}

Esta primera fase del estudio utilizó entrevistas focalizadas en grupo como herramienta de investigación (Lindlof, 1995). Un estudio reciente (López Gómez, 2003) sugiere que el mejor modo de estudiar este particular fenómeno con niños es reunir a pequeños grupos, de 3 ó 4 miembros, para disminuir la intimidación que los niños puedan sentir hacia el investigador y hacia el tema.

\section{Número y tipo de informantes}

Se reunió una muestra por conveniencia. Se entrevistaron 30 niños de 10 a 12 años (15 nińas y 15 niños), estudiantes de cada tipo de escuela (pública y privada) en cada una de las ciudades capitales de los tres estados que conforman el noreste del país: Ciudad Victoria, Tamaulipas; Monterrey, Nuevo León y Saltillo, Coahuila ( $\mathrm{n}=180)$.

Las entrevistas fueron grabadas en audio y transcritas para su interpretación y el diseño de la siguiente fase del estudio.

\section{Resultados}

Procesos de socialización (redes de comunicación interpersonal e instituciones que colaboran en los procesos de socialización política).

En Ciudad Victoria, Tamaulipas, al igual que en Saltillo y Monterrey, los niños mencionaron estar enterados de que este es un año de elecciones. Dicha información en su mayoría la han obtenido por medio de la televisión y las pláticas entre familia, principalmente con la mamá y/o el papá, pero también con otros miembros de la familia como hermanos, abuelitos o tíos. Los temas de tales conversación reportados fueron quiénes son los candidatos, quién sería el mejor para presidente realizando una comparación con el gobierno actual y sobre la propaganda en las calles, y los periódicos.

Aunque en menor grado que los padres, los maestros también parecen jugar un papel importante en este proceso: Los niños en Saltillo coinciden en que los maestros y en ocasiones hasta la directora hablan frecuentemente de los contenidos televisivos, y de temas relacionados con la política, reportándose casos extremos como el que narra David Isaac al referirse a su maestro:

278 • Enrique Huerta, Berenice Bañuelos, Alejandra Rodríguez, Sandra Ileana, Carmen Gómez 
- En veces nos dice de que él trabaja ahí en el PRI y dice de que ahí tiene cosas que le dan, cosas del PAN, pero él, como es del PRI, se las regala a otras personas.

- ¿Cosas como qué?

- Playeras, gorras, plumas, y las regala porque él es del PRI.

- A ustedes les ha tocado que les regalen algo así?

- $\mathrm{Si}$, nos regaló una vez plumas del PRI.

David Isaac, escuela pública, 11 años.

Por otro lado, se detectó que la comunicación que sostienen los padres con sus hijos ha sido frecuentemente para explicar conceptos; los niños reportaron que no perciben que sus padres y madres hayan tratado de inculcarles opiniones y/o juicios ideológicos.

- Hay veces cuando tu mamá te dice... que está ella platicando y tu así de que "mamá es que mira, yo opino esto" y ella te dice: "Usté cállese, que son pláticas de mayores".

Denisse, escuela privada, 12 años.

A diferencia de Victoria, los niños de Monterrey no reportaron platicar con frecuencia con sus papás sobre política, aunque sí de algún tipo de propaganda electoral. Pese a que en principio reportaron esa aparente falta de discusión política, las concepciones políticas de los niños parecen acercarse a aquellas que perciben en los padres. Por ejemplo:

- Mis papás siempre votan por el PAN, por que por el PRI, lleva muchos años y casi no ha construido muchas colonias, y el PAN tiene poquito y ya cada vez lleva mucho, creo que ya lleva mucho construido, creo que lleva más que el PRI.

Rubén, pública, 12 años.

Lo anterior permite sugerir que el primer referente de los nińos en cuanto a política se refiere, proviene de los comentarios que escuchan de sus padres, y en menor medida, de sus maestros. Pocos niños fueron los que mencionaron comentar lo visto en los noticieros con sus compañeros durante el recreo o en el salón de clases, sin que esto signifique antipatía al tema, simplemente que los niños tienen otros temas con más prioridad en su agenda. 
Exposición a contenidos televisivos relacionados con la politica

(Relación especifica de la televisión y otros medios de comunicación en los procesos de socialización política de los niños)

Los niños obtienen la información en los diversos canales televisivos de los candidatos y partidos políticos, recordando a los tres principales (PAN, PRI, PRD), sin embargo en dicha socialización cabe resaltar la participación de la familia, que les permiten ver los diversos noticieros y en algunas ocasiones comentan lo importante que es estar informado. Los niños mencionan que intercambian opiniones e información acerca de la política.

Por otra parte, a pesar de su corta edad, se ha observado en ellos un interés importante por mantenerse informados de la situación política del país. Para obtener esta información ven los noticieros más importantes, mismos que tienen identificados por nombre y hora de transmisión, como el noticiero de Carlos Loret, Lolita Ayala a las 14:30 hrs., Joaquín López Doriga, en el caso de los tres estados, además de que los noticieros locales, que también son ampliamente vistos en Saltillo y Monterrey,

Es relevante destacar que los niños de Ciudad Victoria y Saltillo mencionan que el hecho de que ellos vean noticias se debe a que sus padres las ven cuando descansan por las noches y ellos solos se acercan, o bien como en Monterrey, cuando por las mañanas mientras la madre de familia prepara los alimentos; prenden la televisión para ver las noticias y en ese tiempo las ven. Por otra parte, los niños en general mencionan que sus padres si les permiten que vean las noticias,

$\mathrm{Si}$, mi papá me dice que es bueno ver noticias para que me informe.

Ricardo, Escuela privada, 11 años.

Yo de repente de que sale una noticia no, y pues yo estoy medio dormida desayunando ya de que se empieza a reír y le digo por que te ríes y me dice, es que... ya me empieza a explicar todo y al final empiezo a reírme. Nunca le entiendo a las noticias aunque, bueno cuando me explica mi papá ya les entiendo mejor.

Alejandra, Escuela privada, 10 años.

Para los niños de Saltillo, los programas de comedia y en particular El Privilegio de Mandar, son vistos con regularidad. Los niños tienen el conocimiento de que dichos programas imitan y exageran lo que ocurre en la realidad política nacional, pero hubo algunos casos en los que admitieron que ese programa les informa sobre los eventos actuales.

280 - Enrique Huerta, Berenice Bañuelos, Alejandra Rodriguez, Sandra Ileana, Carmen Gómez 
- ¿Ustedes creen que haya un partido que se preocupe más por la gente?

— Sí, el PRD, ahí 'tá López Obrador, con lo’ vieeeejeeeejiiiiiiito

(risas).

- ¿Cómo saben eso?

- (TODOS) Por el Privilegio de Mandar.

Saltillo, escuela pública, 11 años.

Se observa también, como menciona López Gómez (2003), que los niños prefieren las noticias locales sobre las nacionales. Durante el tiempo en que se realizó la entrevista, los niños saltillenses enfatizaron su preocupación por los mineros atrapados en Sabinas, Coah. y la ausencia del presidente Fox en su Estado en ese momento de crisis. Y en el caso de Monterrey, se observó con el caso Santoy

Ahorita nada más está viendo la (noticia) de los niñeros y la de Riveroll o no sé qué, de Diego Santoy Riveroll.

Monterrey, Daniela, Privada, 10 años.

\section{CONCEPCIONES, VALORES Y ORIENTACIONES POLÍTICAS}

En cuanto a la concepción de un político y lo que este hace los nińos mencionan que son personas elegidas popularmente, para ocupar un puesto determinado donde gobernarán a los ciudadanos. Otros mencionan que los políticos son los que representan a los partidos políticos, destacan que la política si ayuda pero sólo cuando están en campaña que los candidatos ofrecen o dan cosas, prometen y después ya no cumplen.

En cuanto a valores se han creado imágenes positivas y negativas de los políticos en general.

Se tiene una percepción de la política más negativa que positiva, sin embargo los niños tienen conocimiento de la obras que algunos políticos han realizado y otorgan valores como que son buenos, hay algunos malos, etc.

En general la opinión que tienen de Vicente Fox es favorable ya que mencionan que ha realizado obras, ayuda a los niños con computadores en las escuelas, y el programa enciclomedia que han puesto en las escuelas. Asimismo, consideran que Fox ha estado trabajando, pero los diputados y senadores no le han aprobado sus programas para crear cambios. Expresan que existe corrupción en el gobierno. 
Pues también, lo que decimos de la política es que en estos años sí hubo mucho avance con Fox pero también los demás como que no lo dejaron avanzar con el país.

Monterrey, privada, José, 12 años.

José-También que Fox si se preocupa por nosotros y el Peje no, casi no. No he visto que salga en la tele que el Peje hable con niños. A Vicente Fox sí lo he visto que sale que habla con unos y que habla con otros y va a escuelas a hablar con ellos.

Monterrey, pública, José, 12 años.

Se observó que los niños tienden a reproducir sus juicios sobre los personajes según lo que observan en la televisión, ya sea por programas cómicos, por los spots o noticieros.

Con respecto a los candidatos la mayor parte tiene conocimiento de quienes son y a que partidos pertenecen e incluso conocen los sobrenombres, por ejemplo, el más nombrado en Saltillo y Ciudad Victoria era "el peje", así como los puestos en los que se desempeñaban anteriormente a López Obrador como Jefe del D. F., Felipe Calderón como Ex Secretario de Energía del gobierno de Fox. Consideran que los niños si son importantes para los políticos. En el caso de Monterrey el más nombrado era Felipe Calderón en la escuela privada y Madrazo en la escuela pública, de López Obrador conocían y se expresan negativamente de él.

Como el éste ¿̨cómo se llama? Péjele.

Monterrery, Mario, pública, 12 ańos.

En cuanto a los conceptos de democracia, afirman no tener conocimiento acerca de ello, por otra parte por justicia identifican bien en que consiste dan ejemplos acerca de los delitos que se comenten o de la violencia y que deben ser sancionados o castigados quienes lo cometen. Pero hacen referencia también a la igualdad.

Respecto a la libertad está palabra la relacionan con la libertad de expresión y la esclavitud y en Monterrey con salir a jugar, o estar en lugares de su gusto.

Los niños tienen conocimiento acerca de sus derechos y de igual forma manifiestan que estos derechos son tanto para los niños como las niñas.

Algunos niños no tienen idea de lo que es un político, sin embargo existen otros niños que definen al político, por ejemplo en Ciudad Victoria, Eduardo

282 • Enrique Huerta, Berenice Bañuelos, Alejandra Rodríguez, Sandra Ileana, Carmen Gómez 
11 años (como una persona que quiere llegar al poder), Ricardo 11 ańos (Una persona que trabaja en el Gobierno), Daniela 11 años (que hacen leyes), Alejandra 12 años (El que decide) que pone reglas para que el país sea mejor.

También manifiestan ideas negativas sobre lo que hacen los políticos destacando frases como Mayela, de 12 años (algunos son corruptos, narcos, hacen las cosas hasta el último, ya cuando van a terminar), Carlos 11 ańos (Que a veces no actúan) o en la opinión de Carol (tratan de ser algo mejor, pero a veces lo logran y a veces no, ya no se sabe cual es la verdad y cual es la mentira).

Son los que a veces les gusta mucho la competencia que están por otros partidos y a veces cuando se enojan va a matar a muchas personas y se roban dinero.

Monterrey, Andrés, privada, 10 años.

Ubican muy bien a los tres candidatos de los partidos del PRI, PAN y PRD destacando Felipe Calderón, Madrazo y Andrés Manuel López Obrador. Cabe señalar que los niños de Ciudad Victoria piensan que no son importantes para los candidatos porque Ricardo 11 ańos (no pueden contar con nuestro voto). O bien Martín 12 años (porque no podemos votar) Así mismo, señala Imelsi 11 años (debería haber mas materiales y programas para progresar).

Por lo que respecta a los derechos de los niños cabe destacar que los ubican perfectamente como: el derecho a la educación, a un nombre, a la familia, a la salud, a tener un hogar, alimentación y, a no trabajar hasta que tengan mayoría de edad. En contraposición a lo que encontraron Mortimore y Tyrrell (2004), los nińos saltillenses y regiomontanos presentan conocimiento sobre los principales candidatos y partidos, sin que se perciba estrecha relación entre el conocimiento y la actitud (positiva o negativa).

Nuevamente, las respuestas de los niños tienden a centrarse en la política local antes que en la nacional. Conocen los nombres y expresan actitudes sobre el presidente municipal y el gobernador. Como en el caso de Monterrey, donde además confunden a los candidatos presidenciales con los candidatos a la alcaldía.

Mis papás cuando hablan de política dicen va a ganar Madero a Madrazo, cosas así. Monterrey, privada, Karla 10 ańos.

Sin embargo, conforme avanza la edad y el nivel educativo, los niños demuestran más agudeza en sus orientaciones políticas, y valores cívicos. A 
continuación, se enlistan algunas de las respuestas de los nińos de una escuela pública de Saltillo, a la pregunta de si la política es útil:

- Si, porque aprendes cómo ser así... cuando votas, y aparte ya vamos a saber como hacer, porque a nosotros nos va a tocar sacar adelante el futuro de México cuando estemos grandes.

- Y depende de nosotros ser buenos ciudadanos.

— Pues si no sabemos nada. ¡Cómo vamos a vivir en el futuro?

- Yo digo que siempre debemos estar informados.

- Así como los chiquitos, que no les importan las noticias, pero así a partir de los doce años es cuando ya se empiezan a interesar...

- Bueno yo creo que los chiquitos también se deben enterar.

- Bueno, sí se enteran, pero como que no les llama tanto la atención.

- Como a nosotros.

Saltillo, escuela pública, 12 años.

Tal pareciera que las construcciones sociales de los nińos en torno a la política se fortalecen conforme pasa el tiempo y en los planes de estudio de la primaria aparecen temas relacionados con el civismo y la democracia, mismos que son cotejados con el contenido de los medios y comentados en las familias.

Prácticas politicas y competencias civicas

(Plano conceptual al de la intención de participar de la puesta en marcha de sus competencias civicas)

Referente a las practicas políticas los infantes de Ciudad Victoria y Saltillo relatan que si estuvieran en edad de votar lo harían, manifestando que lo harían por el candidato del PAN, asimismo, existe una preferencia menor a este partido por el candidato del PRD quedando en una tercer alternativa el candidato del PRI (Madrazo). A pesar de estar considerada la capital del estado de Tamaulipas como priísta. En cambio en Monterrey la preferencia primero era para el PAN, seguida por el PRI y en último término el PRD.

En Victoria consideran que el partido del PAN es bueno ya que no se han visto afectados por los videos escándalos como otros partidos. La política la relacionan con los temas de corrupción en primer término, seguida de la seguridad y la pobreza. En relación a la participación en la política algunos mencionan que no, porque existe corrupción y les crearía problemas.

284 • Enrique Huerta, Berenice Bañuelos, Alejandra Rodríguez, Sandra Ileana, Carmen Gómez 
En cuanto a los partidos políticos, por la información que ellos reciben de la televisión, expresan que el partido mejor es el PAN, después el PRD, y finalmente el PRI, así mismo mencionan que el que cuenta con más recursos es el PRI por eso existe mucha corrupción.

A la mala imagen que los partidos tienen, hay que agregar que es un tema que les parece aburrido y lejano a algunos niños:

No, no me interesa. Porque salen que los gobernadores que están en junta y no, tardan mucho y me desesperan a mí. Hasta la apago la tele. Sí porque me desesperan. Mejor me salgo a jugar. No, no me interesa.

Monterrey, José, pública,12 años.

Otros niños de Saltillo, sin embargo, aunque coinciden con los de Monterrey y Victoria en el desencanto y desconfianza hacia los partidos, también expresaron gran interés en la política y en el deseo de participar en los comicios. Es de resaltar la aparición de conceptos como la anulación del voto, acción que podría representar otro tipo y otro nivel de participación.

Yo si votaría, pero tacharía todos.

Pero iría a votar.

Pues es que aquí hay muchos que no van a votar, y esos votos, ¿dónde quedan? esos votos van a la basura.

Y luego ellos se quejan porque no querían esos gobernantes.

Que tienes que luchar por lo que quieres. Que si vas a ir a votar, por lo menos va a quedar tu voto ahí, para...

Lo puedes tachar todo, pero fuiste a votar.

Porque si no hay trabajo te tienes que ir a trabajar a otra parte.

Sin embargo están orgullosos de ser mexicanos y algunos mencionaron que México es un país con mucha riqueza cultural en comidas, costumbres y regiones.

A mi si, por que tengo el orgullo de ser mexicano, porque siempre voy a vivir aquí, aquí en México tengo un lugar donde vivir, no me puede correr.

Jesús: a mi también, me gusta mejor ser mexicano

Monterrey, Rubén, pública, 12 años.

El rol de la televisión en la socialización politica de los niños: resultados preliminares $\bullet 285$ 
Es importante destacar que los niños si votarían si pudieran y que lo harían (por el que nos convenga más) Mayela 12 años, (el que tenga mas propuestas para nosotros) Alejandra 12 años, o (el que haga mas porque a veces dicen y no hacen nada) Andrea 12 años.

Por lo que respecta a su interés por los temas políticos en su mayoría se preocupan por solucionar la corrupción, la pobreza y la violencia y mencionan algunos problemas locales.

En su mayoría a los niños les gustaría participar en la política porque podrían ayudar a mucha gente, hacer más escuelas, moderar la contaminación, la tala inmoderada, sin embargo, cabe destacar que a otro grupo de niños no les gustaría participar en política porque...

Te hacen fraude, o les hacen cosas, que no creo que se las busquen o bien, si tú no haces las cosas la gente te dice un chorro de cosas.

\section{Discusión}

Estos hallazgos preliminares apuntan al gran peso que tiene la televisión como agente socializador en la niñez, luego de las otras dos instancias socializadoras primordiales: Los papás y los maestros.

La televisión ha permitido a los nińos crear una imagen determinada de la situación política que impera en el país así como también percibir la problemática de la misma. Sin embargo, los hallazgos parecen indicar que esta información, para que sea asimilada en los nińos, debe ser reforzada por comentarios de los padres y los maestros, quienes moldean e inscriben las actitudes que con el paso del tiempo reproducirán los niños.

Es muy importante destacar que los nińos de las escuelas privadas parecen estar mayormente expuestos a los contenidos televisivos lo que provoca que en su mayoría estén informados de los procesos políticos que se llevan a cabo en el país. Así mismo, efectúan análisis sobre los diversos actores políticos y de su papel ante la sociedad emitiendo juicios a veces muy duros pero que son parte de la realidad que les rodea.

Por otro lado, se observa que hay un gran sentido de responsabilidad hacia las diversas problemáticas que se viven actualmente en el país y disposición por participar con acciones claras en beneficio de los que más lo necesitan.

Otro aspecto a destacar es el alto nivel de orgullo por ser mexicanos, valor que puede darles una identidad firme como futuros ciudadanos y posibles actores políticos.

286 Enrique Huerta, Berenice Bañuelos, Alejandra Rodríguez, Sandra Ileana, Carmen Gómez 
De lo anterior podría desprenderse un modelo explicativo del proceso socializador de los niños:

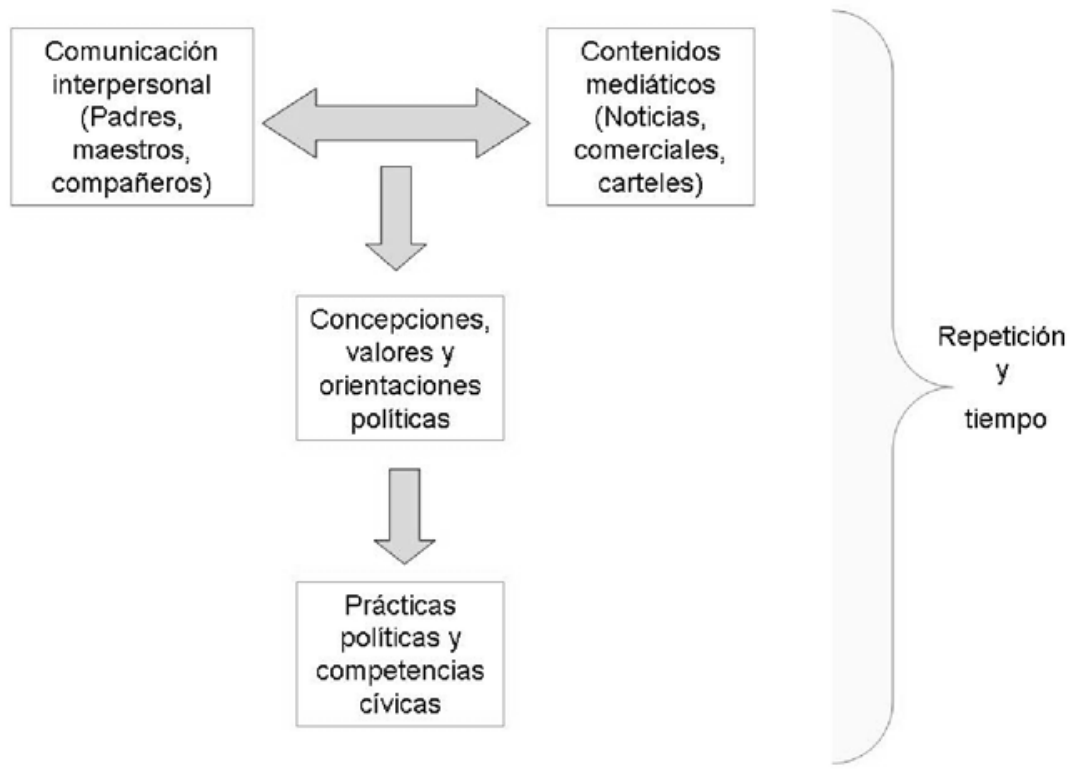

Una investigación de índole cualitativa será la continuación del presente estudio, en la que se procurará cubrir las cuatro esferas mencionadas en el cuadro anterior de tal modo que se permitan hacer generalizaciones sobre el grupo de los niños y niñas de doce años en el noreste del país.

\section{BiBLIOGRAFÍA}

Buckingham, D. (2000). The making of citizens: Young people, news and politics. Londres y Nueva York: Routledge.

Eveland, W., McLeod, J., Horowitz, E. Communication and age in childhood political socialization: an interactive model of political development. (1998). Journalism and Mass Communication Quarterly, 75 (4), pp.699-719.

Gunter, B. y McAleer, J. (1997). Children and Televisión. Nueva York: Routledge. Ibarra, A. (2003). Televisión y socialización politica de escolares en la zona metropolitana (Tesis de doctorado, Universidad de Guadalajara).

Kiousis, S. McDevitt, M., Wu, X. (2005). The Genesis of Civic Awareness: Agenda Setting in Political Socialization. Journal of Communication, pp. 55, 756774.

El rol de la televisión en la socialización politica de los niños: resultados preliminares $\bullet 287$ 
Lindlof, T. (1995). Qualitative research methods. Thousand Oaks: Sage.

López G., G. (2003). Televisión y socialización politica de los niños en Monterrey Juchitán. Tesis de Maestría, Tecnológico de Monterrey, Campus Monterrey.

Mortimore, R. y Tyrrell, C. (2004). Children's acquisition of political opinions, Journal of Public Affaire, .4 (3), pp.279-299.

SEGOB (2002, agosto). Conociendo a los ciudadanos mexicanos: principales resultados de la Encuesta Nacional sobre Cultura Política y Prácticas Ciudadanas 2001. Este País, (137), pp.1-24.

Segovia, R. (1975). La socialización politica del niño mexicano. México: El Colegio de México

Tapia, E. (2003). Socialización Politica y educación cívica en los niños. Querétaro, México: Instituto Mora.

Sears, D. O., \& Valentino, N. A. (1997). Politics matters: Political events as catalysts for preadult socialization, American Political Science Review, 91, 45-65.

Weintraub, E. y Pinkleton, B. (2001). The role of parental mediation in the political socialization process, Journal of Broadcasting and Electronic Media, 45 (2), pp.221-241.

288 • Enrique Huerta, Berenice Bañuelos, Alejandra Rodríguez, Sandra Ileana, Carmen Gómez 